Numer i cal anal ysi s of suppressi on effect s on opt i cal feedback noi se by super posi t i on of hi gh frequency current in sem conduct or I asers

\begin{tabular}{|l|l|}
\hline 著者 & I m an Sazzad MS. , Yamada M nor u \\
\hline $\begin{array}{l}\text { j our nal or } \\
\text { publ i cat i on t i t I e }\end{array}$ & I EEE J our nal of Quant um El ect r oni cs \\
\hline vol une & 49 \\
\hline number & 2 \\
\hline page $r$ ange & $196-204$ \\
\hline year & $2013-01-01$ \\
\hline URL & ht t p: //hdl . handl e. net /2297/34122 \\
\hline
\end{tabular}




\title{
Numerical Analysis of Suppression Effects on Optical Feedback Noise by Superposition of High Frequency Current in Semiconductor Lasers
}

\author{
Sazzad M.S. Imran and Minoru Yamada, Fellow, IEEE
}

\begin{abstract}
Semiconductor lasers tend to be suffered by the optical feedback (OFB) noise caused by reflection of the output light at surface of the optical disc or the optical fiber. Superposition of high frequency (HF) current is used as a technique to suppress the OFB noise. However, this is not effective when frequency of the $\mathrm{HF}$ current coincides with a rational number of the round trip time for the OFB. This paper shows numerical simulations on the phenomena of the OFB noise, its suppression by the superposition of HF current and conditions at which the HF current was unable to suppress the noise. The model used here was based on multimode rate equations that include non-linear gain, Langevin noise sources, the OFB and the HF superposition. Generating mechanism of the OFB noise and its suppression are explained with approximated but analytical equations. Excellent correspondence between experimental data and simulation is also demonstrated.
\end{abstract}

Index Terms - Optical feedback noise, semiconductor laser, mode hopping, high frequency current, intensity noise, modulation.

\section{INTRODUCTION}

S EMICONDUCTOR lasers play a central role in the growing world of optoelectronic technologies. A measure of the importance of this emerging optoelectronic technology is provided by the optical disc players and the optical fiber communication system. It has been recognized that optical feedback (OFB), which is induced by the re-injection of output light into the laser followed by reflection at the surface of connecting optical device, causes excess noise in the output of the laser [1]. This excess noise is called OFB noise. Experiments show that the noise level is increased by $20 \mathrm{~dB}$ or more as a result of the optical feedback [2]. The increase of noise degrades performance of the system.

Intense research activity has been focused on the suppression of the OFB noise of the lasers [3]-[10]. Superposition of high-frequency (HF) current is the most

Manuscript received October 19, 2012; accepted December 17, 2012

S.M.S. Imran and M. Yamada are with the Division of Electrical and Computer Engineering, Graduate School of Natural Science and Technology, Kanazawa University, Kakuma-machi, Kanazawa 920-1192, Ishikawa, Japan (e-mail: imran@stu.kanazawa-u.ac.jp;myamada@t.kanazawa-u.ac.jp). popularly used method to suppress OFB noise. The OFB noise is well suppressed by suitable selections of frequency and amplitude of the superposed current. However, it has been experimentally reported that the OFB noise is not suppressed when frequency of the superposed current and round-trip frequency of the OFB are in relation of rational numbers [10]. Author's group of this paper gave a theoretical analysis on this problem based on mode competition phenomena among external cavity modes which are built in the space between laser front facet and the reflecting point of OFB [10]. Since author's previous analysis was based on small signal approximation, quantitative assessment for conditions unable to suppress OFB noise was difficult.

In this paper, we present a new model by which generation of the OFB noise and its suppression by the superposition of $\mathrm{HF}$ current can be numerically simulated. Conditions unable to suppress the OFB noise are evidently shown.

Our model was applied to $850 \mathrm{~nm}$ GaAs lasers, and characteristics of the OFB noise are expressed in terms of relative intensity noise (RIN).

In the next section, model of analysis and basic equations are derived. In Sec. III, procedure of numerical calculation is explained. In Sec. IV, characteristics of the OFB noise without superposition of the HF current are examined to understand the mechanism of noise generation. In Sec. V, ability of noise suppression by help of superposition of the HF current is demonstrated. In Sec. VI, mechanism of the noise suppression is explained based on approximated equations. In Sec. VII, situations unable to suppress the noise are examined based on calculated data. In Sec. VIII, conclusions are given.

\section{Model of ANALysis}

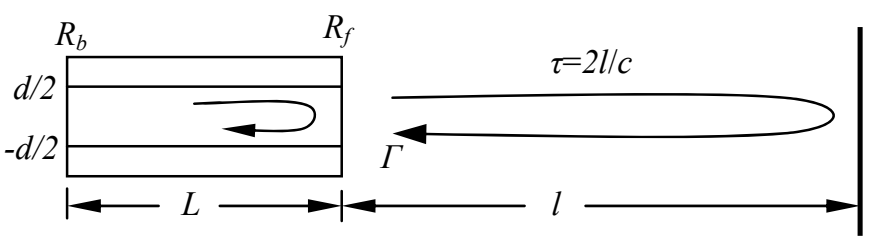

Fig. 1: Operation of a semiconductor laser under optical feedback. 
Operation of a semiconductor laser under OFB is illustrated in Fig. 1, where output light from the laser is reflected back from an external mirror and re-injected into the laser. The cavity length and the effective refractive index of the laser are $L$ and $n_{r}$, respectively. The distance between the laser and the external mirror is $l$. Power reflectivity of the front facet is $R_{f}$. Ratio of the OFB to the front facet is $\Gamma$. The round-trip time is $\tau=2 l / c$, where $c$ is the speed of light in vacuum.

The electric component of the lasing field is described as follows.

$$
E(\mathbf{r}, t)=\sum_{p} \widetilde{E}_{p}(t) \Phi_{p}(\mathbf{r}) \exp \left(j \omega_{p} t\right)+c . c .
$$

where $p=0, \pm 1, \pm 2, \pm 3, \ldots$ is an index to indicate each longitudinal mode of the solitary laser, $\widetilde{E}_{p}(t)$ is a slowly time-varying complex amplitude which is defined with an optical phase $\theta_{p}(t)$ as

$$
\widetilde{E}_{p}(t)=\left|\widetilde{E}_{p}(t)\right| \exp \left(j \theta_{p}\right)
$$

$\Phi_{p}(\mathbf{r})$ is a field spatial distribution function normalized as

$$
\int_{0}^{L} \int_{-\infty}^{\infty} \int_{\infty}^{\infty}\left|\Phi_{p}(\boldsymbol{r})\right|^{2} d x d y d z=1
$$

We suppose here that the transverse mode is stable fundamental mode.

Electric field amplitude $\left|\widetilde{E}_{p}(t)\right|$ can be transformed to photon number $S_{p}(t)$ using the following relationship postulated by quantization of the lasing field [12]

$$
2 \varepsilon\left|\widetilde{E}_{p}\right|^{2}= \begin{cases}\left(S_{p}+1\right) \hbar \omega & \text { for optical emission } \\ S_{p} \hbar \omega & \text { for optical absorption }\end{cases}
$$

where $\varepsilon$ is the dielectric constant of the active region.

Based on above mentioned notations, the rate equations for the modal photon number $S_{p}(t)$, modal phase $\theta_{p}(t)$ and number of injected electrons $N(t)$ can be obtained as [13-16]

$$
\begin{gathered}
\frac{d S_{p}}{d t}=\left(G_{p}-G_{\text {tho }}+\frac{c}{n_{r} L} \ln \left|U_{p}\right|\right) S_{p}+\frac{a \xi N / V}{\left[2 \frac{\left(\lambda_{p}-\lambda_{0}\right)}{\delta \lambda}\right]^{2}+1}+F_{S p}(t) \\
\frac{d \theta_{p}}{d t}=\frac{1}{2}\left[\frac{\alpha a \xi}{V}(N-\bar{N})-\frac{c}{n_{r} L} \varphi\right]+F_{\theta_{P}}(t) \\
\frac{d N}{d t}=-\sum_{p} A_{p} S_{p}-\frac{N}{\tau_{S}}+\frac{I}{e}
\end{gathered}
$$

where $G_{p}$ is the gain of the mode $p$ whose wavelength is $\lambda_{p}$. $G_{t h o}$ is the threshold gain of the solitary laser. $U_{p}$ is a function counting contribution of the OFB to the instantaneous photon number $S_{p}(t)$ of the mode $p$. These parameters are defined as

$$
\begin{gathered}
G_{p}=A_{p}-B_{p} S_{p}-\sum_{q \neq p}\left(D_{p(q)}+H_{p(q)}\right) S_{q} \\
G_{\text {tho }}=\frac{c}{n_{r}}\left[k+\frac{1}{2 L} \ln \frac{1}{R_{f} R_{b}}\right] \\
U_{p}=1+\left(1-R_{f}\right) \sqrt{\frac{\eta \Gamma}{R_{f}}} \exp \left(-j \omega_{p} \tau\right) \sqrt{\frac{S_{p}(t-\tau)}{S_{p}(t)}} \exp \left(j\left\{\theta_{p}(t-\tau)-\theta_{p}(t)\right\}\right) \\
=\left|U_{p}\right| \exp (-j \varphi) \\
\varphi=-\tan ^{-1} \frac{\operatorname{Im} U_{p}}{\operatorname{Re} U_{p}}+m \pi
\end{gathered}
$$

Here, $A_{p}$ is the linear gain, $B_{p}$ is the coefficient of self-suppression, and $D_{p(q)}$ and $H_{p(q)}$ are the coefficients of symmetric gain saturation and asymmetric gain saturation, respectively. These coefficients are given by [14]

$$
\begin{aligned}
& A_{p}=\frac{a \xi}{V}\left[N-N_{g}-b V\left(\lambda_{p}-\lambda_{0}\right)^{2}\right] \\
& B_{p}=\frac{9}{4} \frac{\hbar \omega}{\varepsilon_{0} n_{r}^{2}}\left(\frac{\xi \tau_{i n}}{\hbar V}\right)^{2} a R_{c v}^{2}\left(N-N_{s}\right) \\
& D_{p(q)}=\frac{4}{3} \frac{B_{p}}{\left(\frac{2 \pi c \tau_{i n}}{\lambda_{p}^{2}}\right)^{2}\left(\lambda_{p}-\lambda_{q}\right)^{2}+1} \\
& H_{p(q)}=\frac{3 \lambda_{p}^{2}}{8 \pi c}\left(\frac{a \xi}{V}\right)^{2} \frac{\alpha\left(N-N_{g}\right)}{\lambda_{q}-\lambda_{p}}
\end{aligned}
$$

In (5), $a$ is the differential gain coefficient, $\xi$ is the field confinement factor, $V$ is the volume of the active region, $\lambda_{0}$ is the peak wavelength and $\delta \lambda$ is the half-width of spontaneous emission. In (6) $\alpha$ is the linewidth enhancement factor and $\bar{N}$ is the time average value of $N(t)$. In (7), $\tau_{s}$ is the electron lifetime, $I$ is the injection current and $e$ is the electron charge. In (9), $k$ is the internal loss in the laser cavity. In (10), $\eta$ is the coupling coefficient into the active region, $\Gamma$ is the optical feedback ratio to the laser cavity, $\omega_{p}=2 \pi c / \lambda_{p}$ is the angular frequency of mode $p, \omega_{p} \tau$ is the phase delay of the field in the roundtrip time. In (12)-(15), $N_{g}$ is the electron number at transparency, $b$ is the width of the linear gain coefficient, $\hbar$ is the reduced Planck constant, $\omega=2 \pi c / \lambda_{0}$ is the central angular frequency, $\tau_{i n}$ is the intraband relaxation time, $R_{c v}$ is the dipole moment and $N_{s}$ is the electron number characterizing the self-suppression coefficient.

$F_{S p}(t)$ and $F_{S \theta}(t)$ in (5) and (6) are the Langevin noise sources to indicate generation of the induced instantaneous fluctuations on photon number and phase due to spontaneous emission and the process of recombination. These are well approximated as Gaussian distributions with zero mean values and are given by [16]

$$
\begin{aligned}
& F_{s p}(t)=\sqrt{\frac{V_{S_{p} S_{p}}}{\Delta t} g_{s}} \\
& F_{\theta p}(t)=\sqrt{\frac{V_{S_{p} S_{p}}}{\Delta t}} \cdot \frac{g_{\theta}}{2\left(S_{p}+1\right)} \\
& V_{S_{p} S_{p}}=\left[\frac{a \xi}{V}\left(N+N_{g}\right)+G_{t h o}\right] S_{p}+\frac{a \xi N}{V}
\end{aligned}
$$

We omit a noise term on the electron extinction in this model because it has a negligible effect on the dynamics in calculated results than those for the photon generation [16]. The electron number $N(t)$ suffers sufficient fluctuation from the Langevin noise sources $F_{S p}(t)$ through (5), (7) and (12).

In (16) and (17) $g_{s}$ and $g_{\theta}$ are random number generations in ranges of [14], [16]

$$
-1 \leq g_{s} \leq 1 \text { and }-1 \leq g_{\theta} \leq 1
$$

and $\Delta t$ is the time-step of the calculation.

The central mode, $p=0$ with wavelength $\lambda_{0}$, is assumed to lie at the centre of the spectrum of gain. The wavelength of the other modes can be written as 


$$
\lambda_{p}=\lambda_{0}+p \Delta \lambda=\lambda_{0}+p \frac{\lambda_{0}^{2}}{2 n_{r} L} \quad p=0, \pm 1, \pm 2, \pm 3, \ldots
$$

where $\lambda_{0}^{2} /\left(2 n_{r} L\right)$ is the longitudinal-mode spacing of solitary laser.

In this paper, effect of the OFB is counted as variation of the equivalent threshold level given by $\left(c / n_{r} L\right) \ln \left|U_{p}\right|$ in (5) and difference of the optical phases between the feedback light and the emitting light $\theta_{p}(t-\tau)-\theta_{p}(t)$ in (6), (10) and (11). Counting manner of the phase difference $\theta_{p}(t-\tau)-\theta_{p}(t)$ is an alternative method for counting the external modes built between the laser facet and the reflecting point with distance $l$ [2], [10]. In case of numerical simulation, counting of the phase difference is more direct analysis than that of the external modes. Therefore, we do not use the idea of the external modes in this paper. The word 'mode' in this paper indicates the longitudinal mode in the solitary laser.

The effect of high-frequency injection is included by modulating the injection current periodically with a modulation frequency $f_{M}$. The pumping term $I$ in equation (7) then has to be replaced by

$$
I=I_{D}+I_{M} \cos \left(2 \pi f_{M} t\right)
$$

where $I_{D}$ is the bias current, $I_{M}$ is the modulation current and $f_{M}$ is the frequency of sinusoidal modulation.

The rate equations (5)-(7) can be used to obtain the RIN of the diode laser for the modulated signal in the presence of optical feedback by integrating them numerically and calculating the spectrum of intensity fluctuations.

It is to mention that in experiment, the individual modes are not distinguished. Rather, the system performance is governed by the total photon number [3]. The RIN for the total photon number is therefore given by

$$
R I N=\frac{\left\langle S_{\Omega}{ }^{2}\right\rangle}{|\bar{S}|^{2}}
$$

where $S_{\Omega}$ is calculated from the fluctuations $\delta S(t)=S(t)-\bar{S}$ of the total photon number $S(t)=\sum S_{p}(t)$ and $\bar{S}$ is the time average of the total photon number.

The values of intensity fluctuation for modulated signal is then computed in terms of RIN by employing the fast Fourier transform to integrate the discrete version of equation (21) as

$$
R I N=\frac{1}{|\bar{S}|^{2}} \frac{\Delta t^{2}}{T}\left|F F T\left[\delta S\left(t_{i}\right)\right]\right|^{2}
$$

We now describe the corresponding numerical procedure that has been followed before we discuss our results in sections IV-VII in detail.

\section{Procedure of Numerical CAlCulation}

Parameters used in the numerical calculation are listed in Table I. We include thirteen longitudinal modes in our numerical simulations, which are performed using fourth-order Runge-Kutta algorithm to solve the rate equations (5)-(7) [18]. Time-step of integration is set as short as $\Delta t=5 \mathrm{ps}$. This short time step corresponds to a cutoff Fourier frequency of $100 \mathrm{GHz}$ that is high enough to guarantee fine resolution of the OFB induced dynamics. The length of the external cavity was set to $l=12 \mathrm{~cm}$. For the coupling constant we have used $\eta=0.02$ to estimate the optical feedback ratio $\Gamma$.

The integration is first made without OFB for solitary laser from time $t=0$ until the round-trip time $t=\tau$. After $t=\tau$, the OFB is counted by using stored data of $S_{p}(t-\tau)$ and $\theta_{p}(t-\tau)$. Dynamics in the steady states are examined after $t=0.5 \mu \mathrm{s}$, which is long enough for the transients to be died out. The integration is then proceeded over a long period of time $T=2 \mu \mathrm{s}$. This time limit ensures computation of noise as low as $500 \mathrm{kHz}$. RIN is then computed directly from the obtained values of $S(t)=\sum S_{p}(t)$ by employing the FFT to integrate equation (22).

Note that noise on the calculated RIN spectrum is due to the finite duration of computed time-resolved signal due to our impossibility to simulate infinitely long laser outputs. The RIN spectra are averaged over several trajectories to improve numerical accuracy [19]. The spectra are then smoothed by running an adjacent averaging of spectral components.

Table I. Typical parameter values used in numerical simulations for 850-nm GaAs semiconductor laser

\begin{tabular}{|c|c|c|c|}
\hline Symbol & Definition & Value & Unit \\
\hline$a$ & tangential gain coefficient & $2.75 \times 10^{-12}$ & $\mathrm{~m}^{3} \mathrm{~s}^{-1}$ \\
\hline$b$ & $\begin{array}{c}\text { dispersion parameter of the } \\
\text { linear gain spectrum }\end{array}$ & $3 \times 10^{19}$ & $\mathrm{~m}^{3} \mathrm{~A}^{-2}$ \\
\hline$\left|R_{c v}\right|^{2}$ & $\begin{array}{c}\text { squared absolute value of the } \\
\text { dipole moment }\end{array}$ & $2.8 \times 10^{-57}$ & $\mathrm{C}^{2} \mathrm{~m}^{2}$ \\
\hline$\delta \lambda$ & $\begin{array}{c}\text { half-width of spontaneous } \\
\text { emission }\end{array}$ & 23 & $\mathrm{~nm}$ \\
\hline$\alpha$ & linewidth enhancement factor & 2.6 & - \\
\hline$\xi$ & confinement factor of field & 0.2 & - \\
\hline$\tau_{i n}$ & $\begin{array}{c}\text { electron intraband relaxation } \\
\text { time }\end{array}$ & 0.1 & $\mathrm{ps}$ \\
\hline$\tau_{S}$ & average electron lifetime & 2.79 & $\mathrm{~ns}$ \\
\hline$N_{S}$ & $\begin{array}{c}\text { electron number characterizing } \\
\text { non-linear gain }\end{array}$ & $1.7 \times 10^{8}$ & - \\
\hline$N_{g}$ & electron number at transparency & $2.1 \times 10^{8}$ & - \\
\hline$V$ & $\begin{array}{c}\text { volume of the laser active } \\
\text { region }\end{array}$ & 100 & $\mu \mathrm{m}^{3}$ \\
\hline$d$ & $\begin{array}{c}\text { thickness of the laser active } \\
\text { region }\end{array}$ & 0.11 & $\mu \mathrm{m}$ \\
\hline$L$ & length of the laser active region & 300 & $\mu \mathrm{m}$ \\
\hline$n_{r}$ & $\begin{array}{c}\text { refractive index of laser active } \\
\text { region }\end{array}$ & 3.6 & - \\
\hline$k$ & internal loss in the laser cavity & 10 & $\mathrm{~cm}^{-1}$ \\
\hline$R_{f}$ & reflectivity of front facet & 0.3 & - \\
\hline$R_{b}$ & reflectivity of back facet & 0.6 & - \\
\hline
\end{tabular}

\section{Generation OF OFB Noise}

In this section we outline the main features of intensity noise in semiconductor lasers which will be important for later discussion of noise reduction. Optical feedback affects the noise and dynamics of the laser in different ways, depending on the strength of external feedback. These different effects can be divided into two regimes of feedback as shown in Fig. 2 for a typical 850-nm GaAs laser [2], [12]. The RIN without feedback has a low value (approximately $10^{-16} \mathrm{~Hz}^{-1}$ ) for frequencies 
below $500 \mathrm{MHz}$ which corresponds to the quantum noise; and shows a broad peak near $3 \mathrm{GHz}$ which corresponds to the relaxation oscillation frequency. The RIN in lower frequency region of the spectrum $(<20 \mathrm{MHz})$ appeared to be enhanced by a large amount $(>20 \mathrm{~dB})$ when the optical feedback ratio $\Gamma$ was increased from 0 to $2.45 \times 10^{-3}$. This RIN enhancement is due to unstable mode hopping between two lasing modes [12]. We call here this type of noise to be low frequency type noise. When OFB ratio was increased more, the RIN profile became flat for wide frequency range from very low frequency to several $100 \mathrm{MHz}$. We call here this type of noise to be flat type noise. This flat-type noise is independent of the mode hopping among lasing modes and is generated due to phase distortion of the lasing modes called the coherence collapse [1], [12].

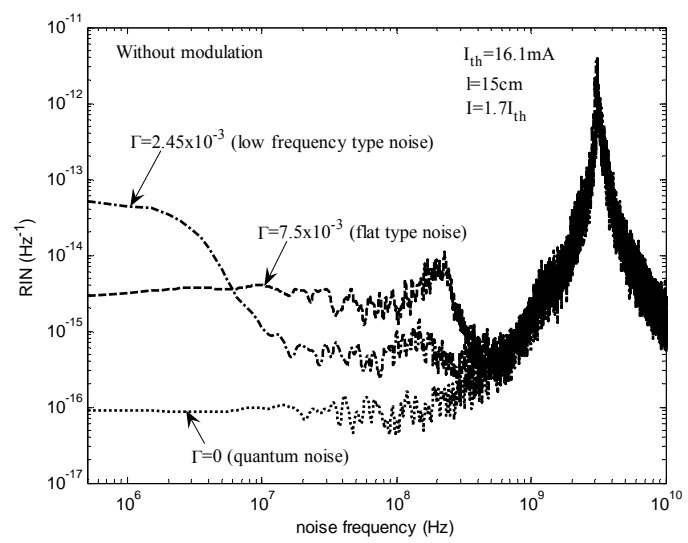

(a)

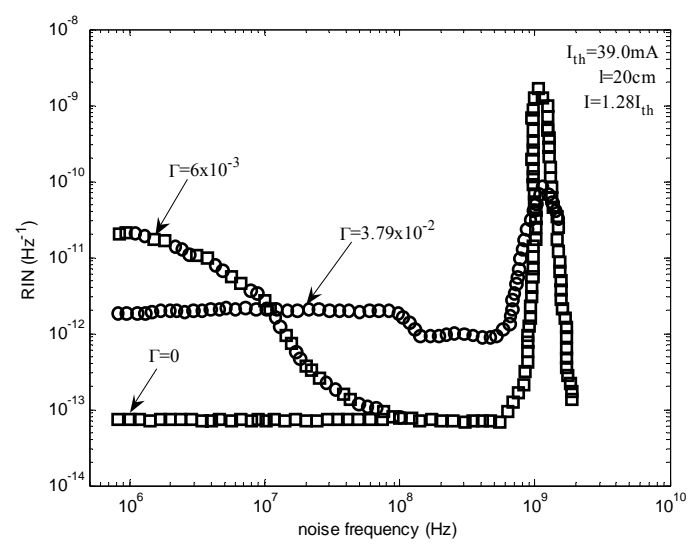

(b)

Fig. 2. Spectra of RIN profiles for different OFB strengths; (a) simulated data, (b) experimental results [2]. The OFB noise is classified into the low frequency type and the flat type based on noise frequency profile [12].

Variation of time averaged modal spectrum is shown in Fig. 3 where (a) is for the operation without optical feedback, (b) is the operation under OFB giving increase of the low frequency type noise and (c) is the operation under OFB giving increase of the flat type noise. The laser shows stable single mode when there is no OFB as shown in (a), but changes to multimode operation by the OFB. However, (b) is not pure multimode operation but unstable mode hopping between two modes.
Since the spectrum is time averaged one, it looks like multimode. (c) is in stable multimode operation.

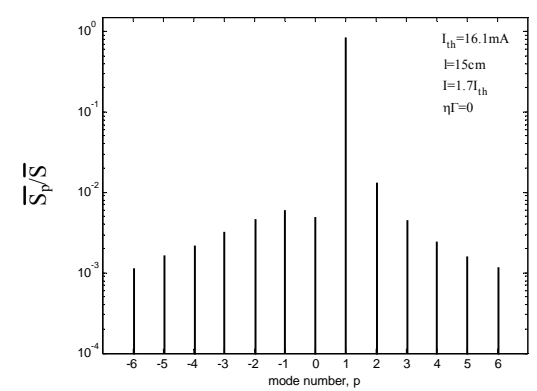

(a)

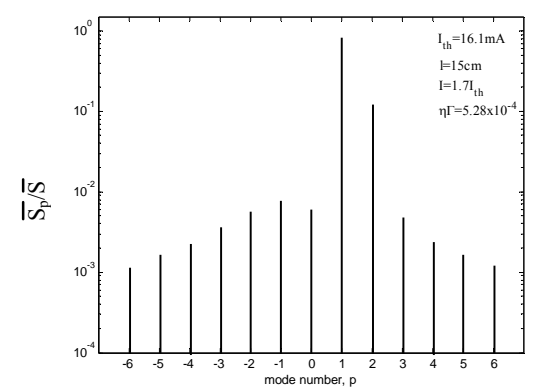

(b)

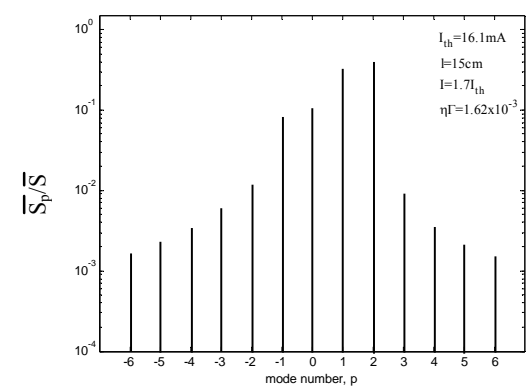

(c)

Fig. 3. Time averaged modal spectrum; (a) without OFB, (b) with OFB for low frequency type noise, (c) with OFB for flat type noise. The operation changes to multimode [(b) \& (c)] from single mode (a) with OFB.

\section{REDUCTION OF OFB NOISE BY SUPERPOSITION OF HF CURRENT}

Calculated examples of noise spectrum of the OFB noise and its suppression by superposition of HF current are shown in Fig. 4 . The feedback distance is $l=12 \mathrm{~cm}$ which corresponds to a round trip time period of $f_{e x}=1 / \tau=c / 2 l=1.25 \mathrm{GHz}$. Feedback strength is $\Gamma=2.45 \times 10^{-3}$ by which the low frequency type noise is enhanced. Frequency of the superposed HF current is $f_{M}=500 \mathrm{MHz}$. Line spectrum in the figure indicates modulation of the photon number with the HF current and its higher harmonics. Quantum noise spectra are also shown for comparison. The noise is increased more than $20 \mathrm{~dB}$ by the OFB, and is well suppressed by introduction of the superposition of HF current. Dependency of suppressed noise level with the modulation depth of HF current is shown in Fig. 5. HF 
modulation of more than $30 \%$ is required to suppress the OFB noise in this numerical example.

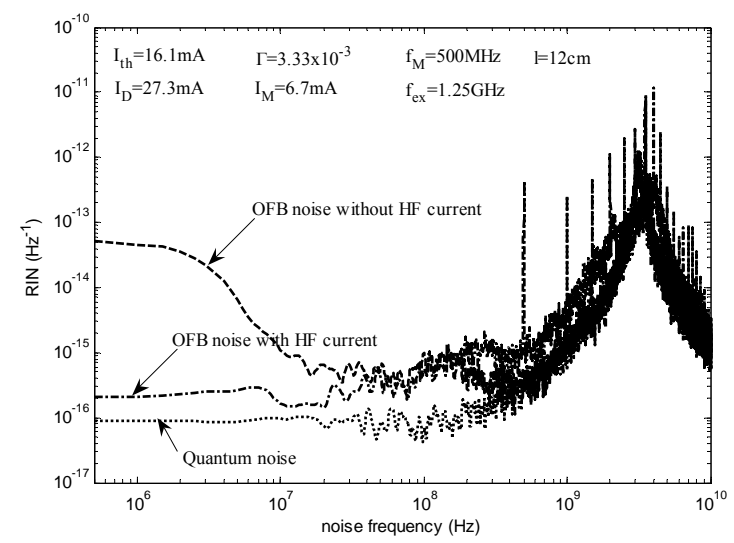

Fig. 4. The simulated spectra of RIN profiles of the OFB noise and suppressed noise by superposition of HF current. The OFB noise is well suppressed by introduction of the HF current.

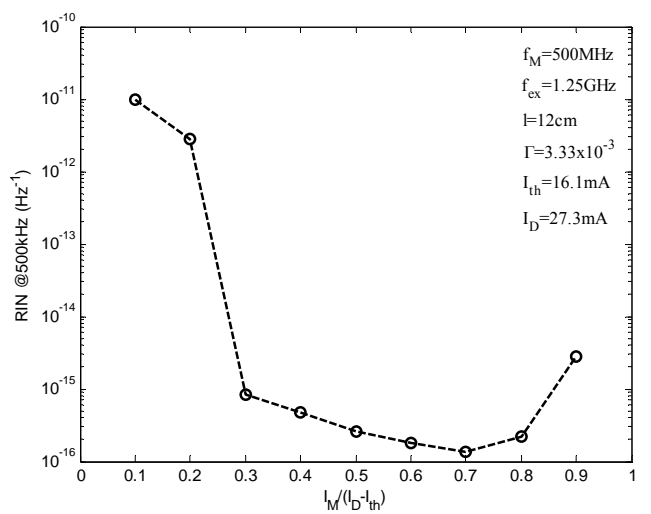

Fig. 5. Dependency of suppressed noise level with the modulation depth of HF current. HF modulation of more than $30 \%$ is required to suppress the OFB noise in this numerical example. Frequency of modulation chosen is $500 \mathrm{MHz}$.

Suppression of the OFB noise by the superposition of HF current is not always effective. Dependency of the modulation frequency $f_{M}$ of the HF current for noise suppression is shown in Fig. 6. The feedback distance is $l=12 \mathrm{~cm}$ which corresponds $f_{e x}=1.25 \mathrm{GHz}$. The RIN is evaluated at the noise frequency of $500 \mathrm{KHz}$. The dashed line indicates the RIN level with neither the OFB nor the superposition of HF current, that is, quantum noise level. The chain line is the RIN level with the OFB but without the HF current. The solid line is the RIN level with the superposition of HF current. The modulation depth is $\mathrm{I}_{\mathrm{M}} /\left(\mathrm{I}_{\mathrm{D}}-\mathrm{I}_{\mathrm{th}}\right)=0.6$ which must be large enough to suppress the OFB noise. The noise is reduced in wide range of the modulation frequency $f_{M}$. However, the RIN raises up when modulation frequency $f_{M}$ of the superposed current coincides with a rational number of the round trip time period $f_{e x}$.

Fig. 7 shows experimental data of variations of the RIN at $1 \mathrm{MHz}$ with the modulation frequency $f_{M}[10]$. The feedback distance is $l=21.4 \mathrm{~cm}$ which corresponds to a round trip time period of $f_{e x}=700 \mathrm{MHz}$. The experimental data shows evidence that the OFB noise raises up when $f_{M}$ and $f_{e x}$ are in rational relations.

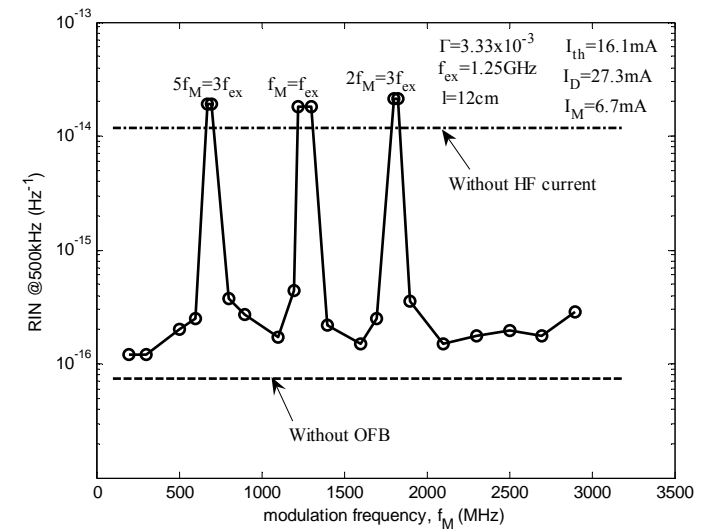

Fig. 6. Calculated data showing dependence of the RIN on modulation frequency of the superposed HF current. The feedback distance is $l=12 \mathrm{~cm}$ which corresponds to $f_{e x}=1.25 \mathrm{GHz}$.

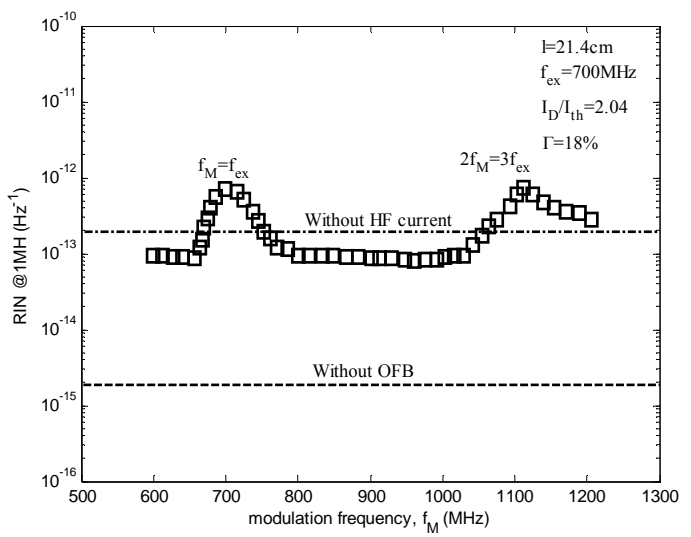

Fig. 7. Experimental data showing dependence of the RIN on modulation frequency of the superposed HF current [10]. The feedback distance is $l=21.4 \mathrm{~cm}$ which corresponds to $f_{e x}=700 \mathrm{MHz}$.

Numerically calculated optical spectrum of the lasing modes are shown in Fig. 8, where (a) is the case that the noise is reduced by the $\mathrm{HF}$ current with modulation frequency of $3 \mathrm{GHz}$, that is, $n f_{M} \neq m f_{e x}$. (b) is the case that the OFB noise raises up with the condition $5 f_{M}=3 f_{e x}$. It seems from the spectra of the internal lasing modes of these figures that the laser operates in multimode for both cases - when the noise is reduced and when the noise is increased.

Temporal variations of lasing modes are shown in Fig. 9. Fig. 9(a) is the case that feedback noise is reduced with condition $n f_{M} \neq m f_{e x}$ corresponding to Fig. 8(a). The lasing modes show stable multimode operation. Fig. 9(b) is the case when the noise raises up with condition $5 f_{M}=3 f_{e x}$ corresponding to Fig. 8(b). The lasing modes show unstable mode hopping between $p=+2$ and $p=+1$. From the optical spectrum, by taking time average value, in Fig. 8(b) it looks as if the laser operates in multimode. 


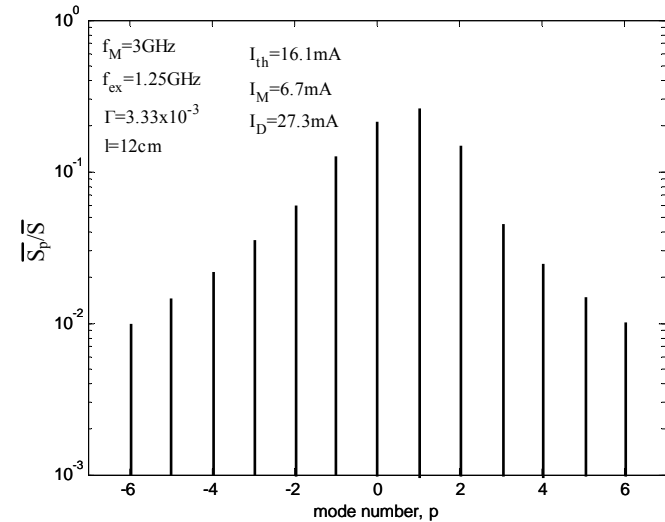

(a)

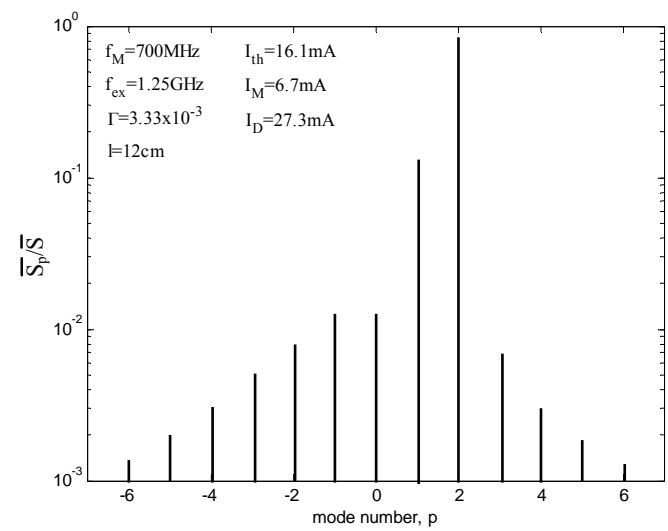

(b)

Fig. 8: Longitudinal mode spectrum with the OFB and HF superposition. (a) is the case that feedback noise was reduced with condition $n f_{M} \neq m f_{e x}$. (b) is the case that the OFB noise raises up with condition $5 f_{M}=3 f_{e x}$.

\section{MECHANISM OF NOISE REDUCTION}

Here, we review generating mechanism of the OFB noise and suppression mechanism by the superposition of HF current.

From equations (5) and (8), the variation of the photon number $S_{p}$ of mode $p$ can be written as,

$$
\begin{aligned}
& \frac{d S_{p}}{d t}=\left(G_{p}-G_{t h o}+C_{p}\right) S_{p}+\frac{a \xi N}{V}+F_{S p}(t) \\
& =\left\{A_{p}-B_{p} S_{p}-\left(D_{p q}+H_{p q}\right) S_{q}-G_{\text {tho }}+C_{p}\right\} S_{p}+\frac{a \xi N}{V}+F_{S p}(t)
\end{aligned}
$$

where

$$
C_{p}=\left(c / n_{r} L\right) \ln \left|U_{p}\right|
$$

represents contribution of the OFB to the lasing mode $p$.

We can obtain a dynamic chart from (23), as shown in Fig. 10 , considering two modes $p$ and $q$. Arrows indicate the flow of the operating point. The lines $L_{p}$ and $L_{q}$ indicate conditions $d S_{p} / d t=0$ and $d S_{q} / d t=0$, respectively. Operation at steady state is at the point $P$ or $Q$. If the operation is at $P$, the laser shows single mode operation with mode $p$. If the operation is at $Q$, the laser shows single mode operation with mode $q$. Selection of the operating point is decided with the initial condition. As found in this figure, the operating points form bi-stable state in a solitary semiconductor laser.

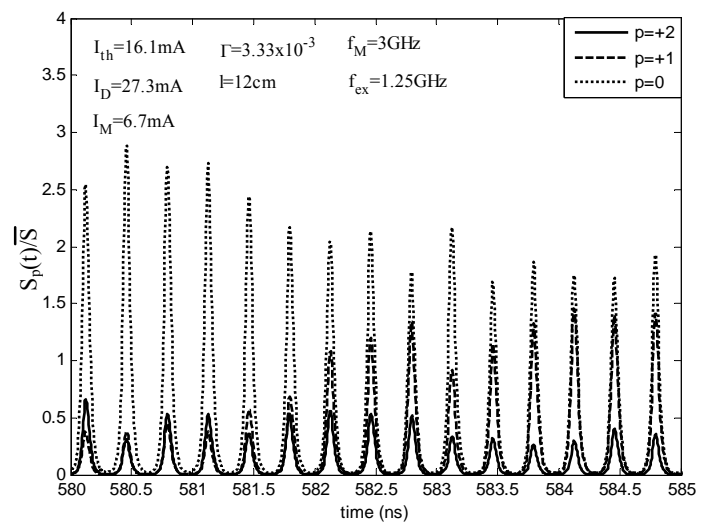

(a)

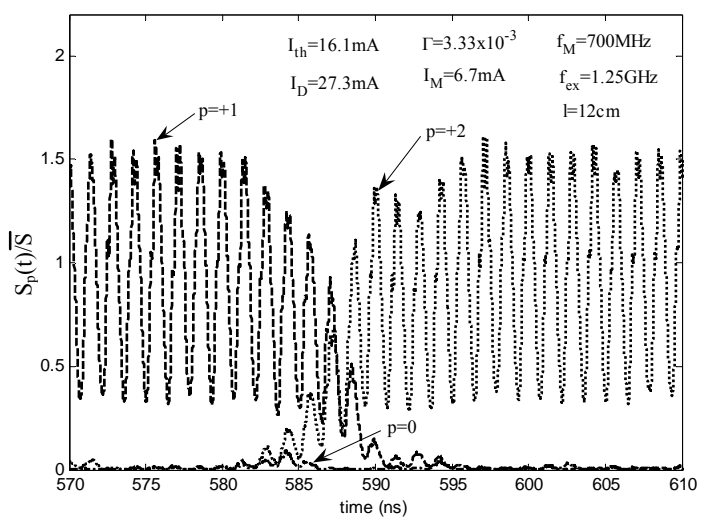

(b)

Fig. 9. Temporal variations of all lasing modes. (a) is the case that feedback noise is reduced with condition $n f_{M} \neq m f_{e x}$ corresponding to Fig. 8(a). The lasing modes show stable multimode operation. (b) is the case when the noise raises up with the condition $5 f_{M}=3 f_{e x}$ corresponding to Fig. $8(\mathrm{~b})$. The lasing modes show unstable mode hopping between $p=+2$ and $p=+1$.

The fluctuating terms $F_{S p}(t)$ and $F_{S q}(t)$ give small movements around the steady point $P$ and $Q$. Since fluctuations are involved in the photon numbers $S_{p}$ and $S_{q}$, and the optical phases $\theta_{p}$ and $\theta_{q}$, the OFB light has also fluctuations. The stronger the OFB, fluctuations in $C_{p}$ and $C_{q}$ become larger through the terms $U_{p}$ and $U_{q}$ defined in (10). Then positions of the lines $L_{p}$ and $L_{q}$ move randomly, resulting in mode hopping between two operating points $P$ and $Q$. Since summed value of the photon number $S_{p}+S_{q}$ is not constant during the mode hopping the laser reveals large variation on the output power.

Now, if we assume the superposition of HF current, that is, the injection current $I$ is modulated with amplitude $I_{M}$ and angular frequency $\Omega=2 \pi f_{M}$,

$$
I=\widetilde{I}+\left(I_{M} e^{j \Omega t}+I_{M}^{*} e^{-j \Omega t}\right) / 2
$$

Corresponding to introduction of the modulation, variations of the electron number $N$ and the photon number $S_{p}$ are expressed as, 


$$
\begin{aligned}
& N=\tilde{N}+N_{M} e^{j \Omega t}+N_{M}^{*} e^{-j \Omega t} \\
& S_{p}=\widetilde{S}_{p}+S_{M p} e^{j \Omega t}+S_{M p}^{*} e^{-j \Omega t}
\end{aligned}
$$

where, $\widetilde{N}$ and $\widetilde{S}_{p}$ are slowly varying terms compared with $\Omega$, and $N_{M}$ and $S_{M p}$ are modulated terms. By substituting eqn. (26) and (27) into (23), the variation in $\widetilde{S}_{p}$ is given by,

$$
\frac{d \widetilde{S}_{p}}{d t}=\left\{\widetilde{A}_{p}-B_{p} \widetilde{S}_{p}-\left(D_{p q}+H_{p q}\right) \widetilde{S}_{q}-G_{t h o}+C_{p}\right) \widetilde{S}_{p}+K_{p}+F_{S p}(t)
$$

with

$K_{p}=\frac{a \xi}{V}\left\{\tilde{N}+N_{M} S_{M p}^{*}+N_{M}^{*} S_{M p}\right\}$

Similar equations can be obtained for $S_{q}$.

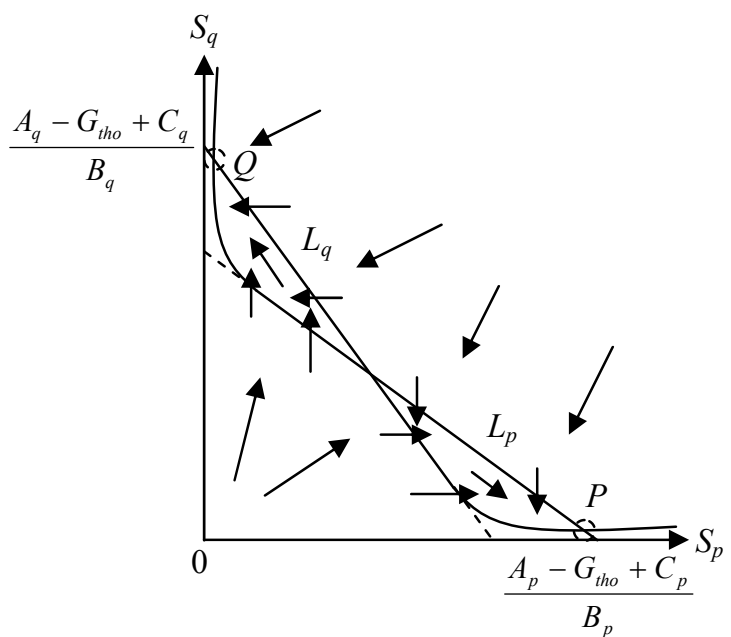

Fig. 10. Dynamic chart indicating mode competition phenomena between two lasing modes. When the OFB level increases the operating point jumps from $P$ to $Q$ or from $Q$ to $P$.

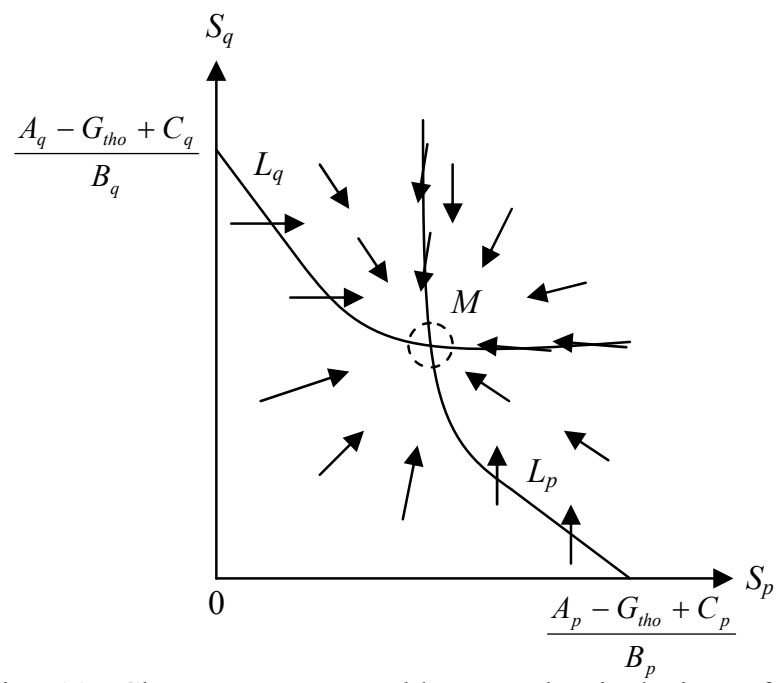

Fig. 11. Change to monostable state by inclusion of HF components in the lasing operation. The operating point $M$ indicates a stable multimode operation of modes $p$ and $q$.

As found from (29), the term $K_{p}$ is increased by the HF modulation when variation of the electron number $N_{M}$ and that of the photon number $S_{M}$ are large enough and in same phase.
With the increase of the term $K_{p}$, the lines $L_{p}$ and $L_{q}$ concave more strongly achieving a monostable state operation as shown in Fig. 11. The point $M$ is an operating point at steady state, which indicates multimode operation of modes $p$ and $q$. In this case of Fig. 11, the operating point slightly moves by the OFB, but never shows the mode hopping. The OFB noise is thus suppressed by the superposition of HF current [7], [10].

\section{INVESTIGATION OF THE CONDITIONS UNABLE TO SUPPRESS NOISE}

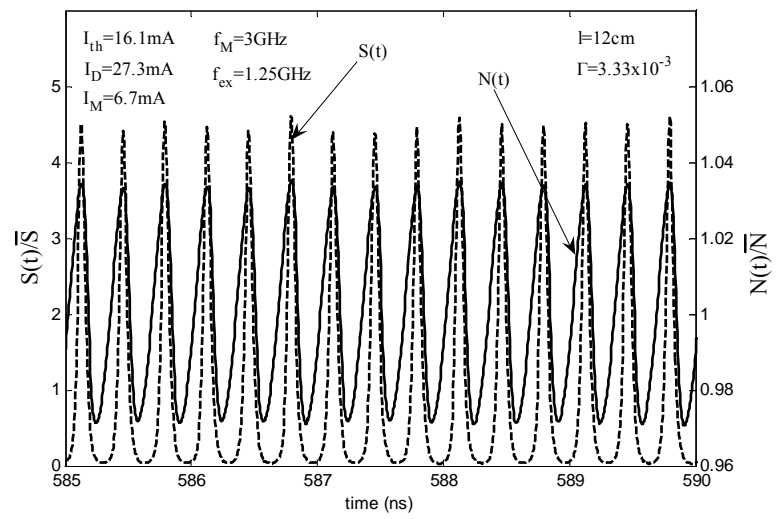

(a)

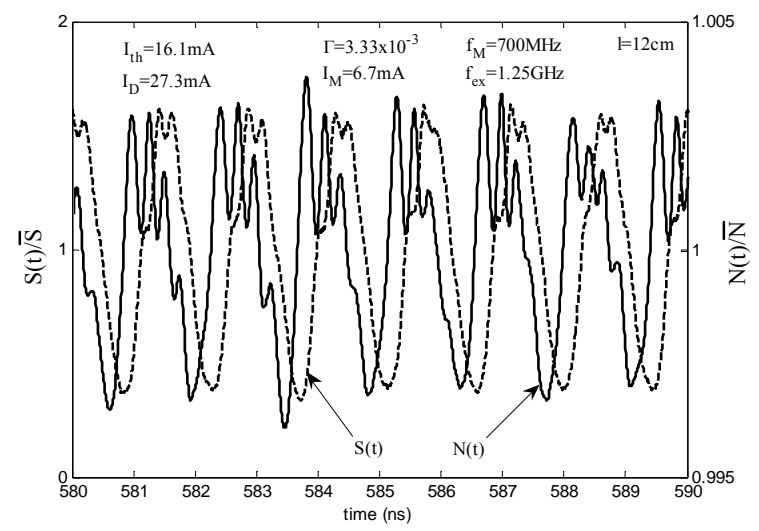

(b)

Fig. 12. Temporal variations of electron number and total photon number. (a) is the case of $n f_{M} \neq m f_{e x}$. Variations of the electron number and the photon number are large enough and are in the same phase. (b) is the case of $5 f_{M}=3 f_{e x}$. Variation of the electron number and that of the photon number have $90^{\circ}$ phase difference. Amplitudes of the variations are small.

As discussed in the previous section, condition to suppress the OFB noise is that, variations of the electron number and the photon number become in the same phase. Calculated values for the temporal variations of the electron number and the total photon number are shown in Fig. 12. (a) is the case of $n f_{M} \neq m f_{e x}$ with which the OFB noise is well suppressed revealing the stable multimode operation. Variations of the electron number and the photon number are large enough and the varying phases between them are same. (b) is the case of $5 f_{M}=3 f_{e x}$ with which the OFB noise is increased with mode hopping remained. 
Variation of the electron number and that of the photon number have $90^{\circ}$ phase difference. Also, amplitudes of the variations are small. Then the term $K_{p}$ in (29) cannot increase in this case.

Temporal variations of the gain $G_{p}$ and the contribution of the OFB $C_{p}=\left(c / n_{r} L\right) \ln \left|U_{p}\right|$ are shown in Fig. 13. (a) is the case of $m f_{M} \neq n f_{e x}$ with which the OFB noise is well suppressed. (b) is the case of $5 f_{M}=3 f_{e x}$ with which the OFB noise is increased with mode hopping remained. We find that the variations of $G_{p}$ and $C_{p}$ are not synchronized when $m f_{M} \neq n f_{e x}$ as shown in (a), but are synchronized with $f_{M}$ having almost $180^{\circ}$ phase difference when $5 f_{M}=3 f_{e x}$ as shown in (b). In the case of $m f_{M} \neq n f_{e x}$, the phase difference between the feedbacked light and emitting light, $\theta_{p}(t-\tau)-\theta_{p}(t)$ in (10), has no fixed relation. Then, variations of the electron number $N$ and the gain coefficient $G_{p}$ are not disturbed by the OFB, resulting in sufficient variation of the photon number $S_{p}$. However, in the case of $m f_{M}=n f_{e x}$, the phase difference $\theta_{p}(t-\tau)-\theta_{p}(t)$ is locked with the rational frequency of $f_{M}$ [20] and works to reduce variation of $G_{p}+C_{p}$ for variations of $G_{p}$ and $C_{p}$ are in inverse phase relation as shown in Fig. 13(b). Then the modulation of the photon number is reduced as found from (5) or (23).

\section{CONCLUSION}

Semiconductor lasers tend to be suffered by the optical feedback (OFB) noise caused by reflection of the output light at surface of the optical disc or the optical fiber. Superposition of high frequency (HF) current is the most popularly used technique to suppress the OFB noise. However, noise suppression by the superposition of HF current does not work when the modulation frequency of the HF current coincides with a rational number of the round trip time for the OFB.

A simulation model giving generation of the OFB noise and its suppression by the superposition of HF current is shown in this paper. Mode competition phenomena among lasing modes in the solitary laser and optical phase delay between the feedbacked light and emitting light were counted in the model together with the Langevin noise sources to explain generation of the OFB noise. The highest OFB noise is caused by the mode competition phenomena among lasing modes which is induced by the OFB.

The superposition of HF current works to modulate both the electron number $N$ and the photon number $S_{p}$, which works to change the operating state from the bi-stable state to the monostable state, and stop the mode hopping resulting in suppression of the OFB noise. However, when the modulation frequency $f_{M}$ of the superposed HF current coincides with a rational number of the returning period $f_{e x}$ of the OFB, modulations of the electron number $N$ and the photon number $S_{p}$ are suppressed by the phase locking effect with undesirable phase relation, resulting in reduced modulation of $N$ and $S_{p}$. Thus the noise suppression effect does not work under the condition $n f_{M}=m f_{e x}$.

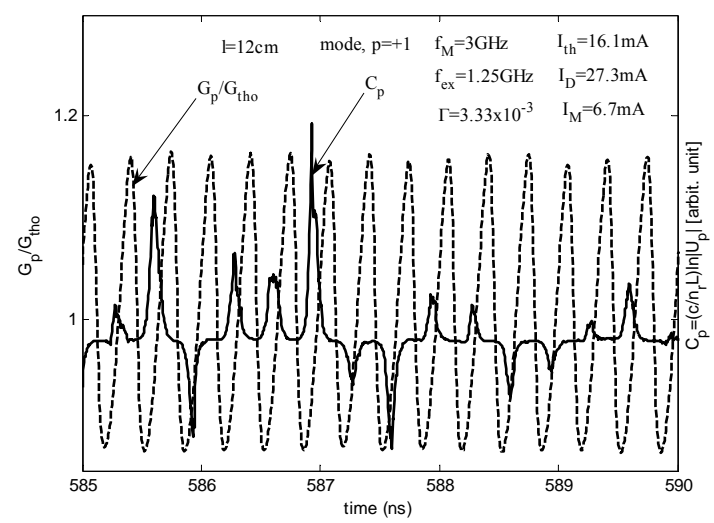

(a)

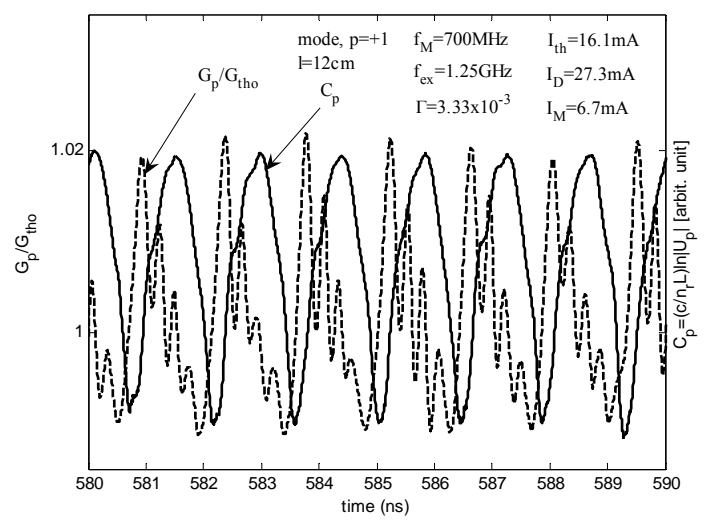

(b)

Fig. 13. Temporal variations of the gain $G_{p}$ and the contribution of the OFB $C_{p}$. (a) is the case of $m f_{M} \neq n f_{e x}$ with which the OFB noise is well suppressed. (b) is the case of $5 f_{M}=3 f_{e x}$ with which the OFB noise is increased with the mode hopping remained. Variations of $G_{p}$ and $C_{p}$ are not synchronized when $m f_{M} \neq n f_{e x}$ as in (a), but are synchronized with $f_{M}$ and have almost $180^{\circ}$ phase difference when $5 f_{M}=3 f_{e x}$ as in (b).

\section{REFERENCES}

[1] K. Petermann, Laser Diode Modulation and Noise, Boston, MA: Kluwer, 1991.

[2] M. Yamada, A. Kanamori and S. Takayama, "Experimental evidence of mode competition phenomena on the feedback induced noise in semiconductor lasers", IEICE Trans. Electron., vol. E79-C, no. 12, pp. 1766-1768, 1996.

[3] A.T. Ryan, G.P. Agrawal, G.R. Gray and E.C. Gage, "Optical-feedback-induced chaos and its control in multimode semiconductor lasers”, IEEE J. Quantum Electron., vol. 30, pp. 668-679, 1994.

[4] J.M. Buldu, F. Rogister, J. Trull, C. Serrat, M.C. Torrent, J. Garcia-Ojalvo and C.R. Mirasso, "Asymmetric and delayed activation of side modes in multimode semiconductor lasers with optical feedback”, J. Opt. B: Quantum Semiclass. Opt., vol. 4, pp. 415-420, 2002.

[5] K. Stubkjaer and M.B. Small, "Feedback-induced noise in index-guided semiconductor lasers and its reduction by modulation”, Electron. Lett., vol. 19, pp. 388-389, 1983. 
[6] E.C. Gage and S. Beckens, "Effects of high frequency injection and optical feedback on semiconductor laser performance", Proc. SPIE, vol. 1316, pp. 199-204, 1990.

[7] M. Yamada and T. Higashi, "Mechanism of the noise reduction method by superposition of high-frequency current for semiconductor injection lasers", IEEE J. Quantum Electron., vol. 27, pp. 380-388, 1991.

[8] A. Ohishi, M. Chinone, M. Ojima and A. Arimoto, "Noise characteristics of high-frequency superposed laser diodes for optical disc systems", Electron. Lett., vol. 20, pp. 821-822, 1984.

[9] J. Sacher, D. Baums, P. Panknin, W. Elsasser and E. O. Gobel, "Intensity instabilities of semiconductor lasers under current modulation, external light injection, and delayed feedback", Physical Review A, vol. 45, no. 3, pp. 1893-1905, Feb. 1992.

[10] M. Yamada, S. Yamamura and T. Okamoto, "Characterization of the feedback induced noise in semiconductor lasers under superposition of high frequency current", IEICE Trans. Electron., vol. E84-C, no. 10, Oct. 2001.

[11] G.P. Agrawal and N.K. Dutta, Semiconductor Lasers, 2nd ed., New York: Van Nostrand Reinhold, 1993.

[12] S.M.S. Imran, M. Yamada and Y. Kuwamura, "A theoretical analysis of the optical feedback noise based on multimode model of semiconductor lasers", IEEE J. Quantum Electron., vol. 48, no. 4, pp. 521-527, April 2012.

[13] M. Ahmed and M. Yamada, "Inducing single-mode oscillation in Fabry-Perot InGaAsP lasers by applying external optical feedback", IET optoelectronics, vol. 4, Iss. 3, pp. 133-141, 2010

[14] M. Ahmed and M. Yamada, "Field fluctuations and spectral line shape in semiconductor lasers subjected to optical feedback", Journal of Applied Physics, vol. 95, no. 12, pp. 7573-7583, June 2004.

[15] S.G. Abdulrhman, M. Ahmed, T. Okamoto, W. Ishimori and M. Yamada, "An improved analysis of semiconductor laser dynamics under strong optical feedback", Journal of selected topics in quantum electronics, vol. 9, no. 5, pp. 1265-1274, Sept./Oct. 2003.

[16] M. Ahmed, M. Yamada and M. Saito, "Numerical modeling of intensity and phase noise in semiconductor lasers", IEEE journal of quantum electronics, vol. 37 , no. 12, pp. 1600-1610, Dec. 2001.

[17] R. Lang and K. Kobayashi, "External optical feedback effects on semiconductor injection laser properties", IEEE J. Quantum Electron., vol. QE-16, pp. 347-355, Mar. 1980.

[18] D. Marcuse, "Computer simulation of laser photon fluctuations: Theory of single cavity laser", IEEE J. Quantum Electron., vol. 20, pp. 1139-1148, Oct. 1984.
[19] D. Lasaosa, M. Vega-Leal and C. Fananas, "Improved time-resolved simulation of amplitude and phase fluctuations in semiconductor laser light”, Journal of Opt. Quant. Electron., vol. 40, pp. 367-372, Apr. 2008.

[20] P. Bak, T. Bohr and M.H. Jensen, "Mode-locking and the transition to chaos in dissipative systems", Physica Scripta, pp. T9: 50-58, 1985 .

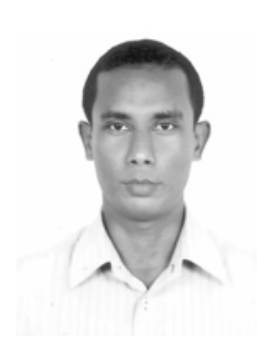

Sazzad M.S. Imran was born in Munshiganj, Bangladesh on November 21, 1978. He received the B.Sc. and M.S. degrees in Applied Physics, Electronics and Communication Engineering from the University of Dhaka, Dhaka, Bangladesh in 2004 and 2006, respectively. He is currently working toward the Ph.D. degree at the Graduate School of Natural Science and Technology, Kanazawa University, Kanazawa, Japan.

In 2007, he joined University of Dhaka as a lecturer and now is on study leave. From 2006 to 2007, he was a lecturer in Electronics and Telecommunication Engineering Department, Daffodil International University, Dhaka, Bangladesh. His research interests are in semiconductor lasers, semiconductor optical amplifiers and optical fiber communication.

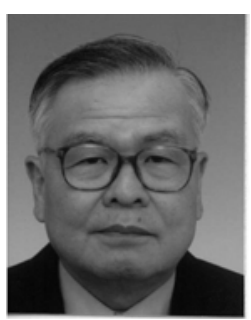

Minoru Yamada (M'82-SM'06-F'10) was born inYamanashi, Japan, on January 26, 1949. He received the B.S. degree in Electrical Engineering from Kanazawa University, Kanazawa, Japan, in 1971, and the M.S. and Ph.D. degrees in Electronics Engineering from Tokyo Institute of Technology, Tokyo, Japan, in 1973 and 1976, respectively.

In 1976, he joined Kanazawa University, where he is currently a Professor. From 1982 to 1983 , he was a visiting scientist at Bell Laboratories, Holmdel, NJ. His current research interests include semiconductor injection lasers, semiconductor modulators, and optical amplifiers utilizing electron beams.

Prof. Yamada received the Yonezawa Memorial Prize in 1975, the Paper Reward in 1976, and the Achievement Award in 1978 from the Institute of Electronics and Communication Engineers of Japan. He is a fellow member of the Japan Society of Applied Physics. 